\title{
Boundary Exponential Stabilization of a One-Dimensional Anti-Stable Wave Equation with Control Matched Disturbance
}

\author{
Ruicheng Li \\ School of Mathematics and Statistics, Shandong Normal University, Jinan, China \\ Email:1621760902@qq.com
}

How to cite this paper: Li, R.C. (2020) Boundary Exponential Stabilization of a One-Dimensional Anti-Stable Wave Equation with Control Matched Disturbance. Engineering, 12, 640-651. https://doi.org/10.4236/eng.2020.129045

Received: September 1, 2020

Accepted: September 19, 2020

Published: September 22, 2020

Copyright $\odot 2020$ by author(s) and Scientific Research Publishing Inc. This work is licensed under the Creative Commons Attribution International License (CC BY 4.0).

http://creativecommons.org/licenses/by/4.0/

\begin{abstract}
In this paper, we are concerned with output feedback stabilization for a one-dimensional anti-stable wave equation with disturbance. First, we design a disturbance estimator for the original system. Then, we propose an output feedback controller for the original system. By calculation, the closed-loop of original system is proved to be exponentially stable and well-posed. Finally, this paper is summarized.
\end{abstract}

\section{Keywords}

Anti-Stable Wave Equation, Disturbance Estimator, Exponential Stabilization, Output Feedback

\section{Introduction}

The wave equation is a set of differential equations derived from Maxwell's equations, which describes the wave characteristics of electromagnetic field. It is an important partial differential equation and has important research significance in the field of control. In recent years, anti-stable one-dimensional wave equation with boundary disturbance has been researched in different ways in the field of control. On this issue, there is used the Lyapunov function approach to design controller in Guo [1] (2014). Besides, active disturbance rejection control (ADRC) has established itself as a powerful control technology in dealing with vast uncertainty in control system. A class of nonlinear systems is dealt with a modified nonlinear extended state observer (ESO) of a time-varying gain in active disturbance rejection control (ADRC) in Zhao [2] (2015). In Wu [3], they apply the active disturbance rejection control (ADRC), an emerging control technology, to output feedback stabilization for a class of uncertain multi-input 
multi-output (MIMO) nonlinear systems with vast stochastic uncertainties. Boundary stabilization is considered for a multi-dimensional wave equation with boundary control matched disturbance that depends on both time and spatial variables. The active disturbance rejection control (ADRC) approach is adopted in investigation in Guo [4]. An algorithm with the active disturbance rejection control approach is developed to reject time and spatially varying boundary disturbances from a multidimensional Kirchhoff plate via boundary control in [5] (2014). A vital step toward ADRC is to estimate the disturbance through an extended state observer (ESO). In Zhao [6] (2015) and [7] (2011), a nonlinear ESO is designed for a kind of lower triangular nonlinear systems with large uncertainty. A nonlinear extended state observer (ESO) is investigated that constructed from piece-wise smooth functions consisted of linear and fractional power functions in Zhao [8] (2015). In Wu [9], they construct a nonlinear ESO for a class of uncertain lower triangular nonlinear systems with stochastic disturbance and show its convergence, where the total disturbance includes internal uncertain nonlinear part and external stochastic disturbance. Besides, the disturbance is then compensated in feedback loop by its estimate. In Guo [10] (2015), the active disturbance rejection control (ADRC) approach is adopted in investigation. In addition, the $\mathrm{ADRC}$ can also efficiently reduce the control energy in practice [11]. Moreover, there are many kinds of disturbances, the "backstepping" method for the problem of stabilization of one-dimensional wave equation with input harmonic disturbance is adopted in the design of the adaptive regulator in Guo [12] (2013). And there are many design methods for a boundary controlled one-dimensional wave equation with external disturbance. A new method is proposed to estimate the total disturbance without using high gain in Zhou [13] (2018). In particular, the derivative of disturbance isn't longer commanded to be bounded, instead, we should relax the disturbance to be $d \in L^{\infty}(0, \infty)$ or $d \in L^{2}(0, \infty)$. The requirement is different from that in the ADRC of lumped parameter systems by high gain [14] [15] [16] [17].

Our main focus is on stabilization for the following anti-stable one-dimensional wave equation with general boundary disturbance:

$$
\left\{\begin{array}{l}
u_{t t}(x, t)=u_{x x}(x, t), x \in(0,1), t>0 \\
u_{x}(0, t)=-q u_{t}(0, t), t \geq 0, \\
u_{x}(1, t)=d(t)+U(t), t \geq 0, \\
u(x, 0)=u_{0}(x), u_{t}(x, 0)=u_{1}(x), x \in[0,1], \\
y_{0}=\left\{u(0, t), u(1, t), u_{t}(0, t)\right\}, t \geq 0,
\end{array}\right.
$$

where $y_{0}$ is the output (measurement), $U(t)$ the input (control), $\left(u_{0}, u_{1}\right)$ the initial value, $q>0, q \neq 1$, and $d \in L^{\infty}(0, \infty)$ or $d \in L^{2}(0, \infty)$ which generally represents an unknown external disturbance. The observer design for system (1.1) can describe the measurement of knocking in the combustion process of automotive engine [18]. For the presence of external disturbance, this system became unstable. A state feedback control was designed in [16]. Next, we con- 
sider system (1.1) in the state space $H=H^{1}(0,1) \times L^{2}(0,1)$. This is enough to show in Feng [19] (2017), it is obvious that using three output signals in his paper. Besides, five equations are used in his study of stabilization and tracking problems, including three-wave equations and two transport equations in the recent work Zhou [20] (2017). There exists the approach of "backstepping" in designing controller. Compared with the method he studied, I simplify it. In this paper, I reduce the number of applying dynamic compensators, using threewave equations and a transport equation to solve the problem. In this paper, we use a new approach to disturbance estimation by directly designing an infinite-dimensional disturbance estimator with using two measurements. Specifically, the stability of closed-loop system is proved by the method of matrix change, which is in the process of innovation.

The paper is organized as follows. In Section 2, we design a disturbance estimator for original system (1.1). The new system is constructed by the known system, and then the disturbance estimator is designed. In Section 3, we are devoted to designing the output feedback control, and proving the exponential stability of the resulting closed-loop system. Finally, gives the concluding remarks.

\section{Disturbance Estimator Design}

In this section, we devote to design a disturbance estimator for system (1.1). To this end, we first introduce the following coupled system consisting of transport equation and wave equation

$$
\left\{\begin{array}{l}
w_{t}(x, t)+w_{x}(x, t)=0, \\
w(0, t)=-\frac{q+c_{0}}{1+c_{0}} u(0, t), \\
\beta_{t t}(x, t)=\beta_{x x}(x, t), \\
\beta_{x}(0, t)=c_{0} \beta_{t}(0, t)+c_{1} \beta(0, t)+c_{1} u(0, t)+c_{1} w(0, t), \\
\beta_{x}(1, t)=0, \\
w(x, 0)=w_{0}(x), \beta(x, 0)=\beta_{0}(x), \beta_{t}(x, 0)=\beta_{1}(x)
\end{array}\right.
$$

where $c_{0}, c_{1}>0$ are tuning parameters.

System (2.1) is known since the injection $u(0, t)$ is one of the original system (1.1).

Let $z(x, t)=u(x, t)+w(x, t)+\beta(x, t)$. Then $z$ is governed by the following wave equation.

$$
\left\{\begin{array}{l}
z_{t t}(x, t)=z_{x x}(x, t), x \in(0,1), t>0 \\
z_{x}(0, t)=c_{0} z_{t}(0, t)+c_{1} z(0, t), t \geq 0 \\
z_{x}(1, t)=U(t)+d(t)-w_{t}(1, t), t \geq 0, \\
z(x, 0)=z_{0}(x)=u_{0}(x)+w_{0}(x)+\beta_{0}(x), x \in[0,1], \\
z_{t}(x, 0)=z_{1}(x)=u_{1}(x)+w_{0}^{\prime}(x)+\beta_{1}(x), x \in[0,1],
\end{array}\right.
$$

We construct an auxiliary dynamic system as follows: 


$$
\left\{\begin{array}{l}
\hat{z}_{t t}(x, t)=\hat{z}_{x x}(x, t), x \in(0,1), t>0 \\
\hat{z}_{x}(0, t)=c_{0} \hat{z}_{t}(0, t)+c_{1} \hat{z}(0, t), t \geq 0, \\
\hat{z}_{x}(1, t)=U(t)-w_{t}(1, t), t \geq 0, \\
\hat{z}(x, 0)=\hat{z}_{0}(x), \hat{z}_{t}(x, 0)=\hat{z}_{1}(x), x \in[0,1],
\end{array}\right.
$$

The error between systems (2.2) and (2.3), $\tilde{z}(x, t)=z(x, t)-\hat{z}(x, t)$, is governed by

$$
\left\{\begin{array}{l}
\tilde{z}_{t t}(x, t)=\tilde{z}_{x x}(x, t), x \in(0,1), t>0 \\
\tilde{z}_{x}(0, t)=c_{0} \tilde{z}_{t}(0, t)+c_{1} \tilde{z}(0, t), t \geq 0, \\
\tilde{z}_{x}(1, t)=d(t), t \geq 0 \\
\tilde{z}(x, 0)=\tilde{z}_{0}(x), \tilde{z}_{t}(x, 0)=\tilde{z}_{1}(x), x \in[0,1],
\end{array}\right.
$$

Define the state space $H=H^{1}(0,1) \times L^{2}(0,1)$ for system (2.4) with the norm

$$
\|(f, g)\|_{H ; \mid \cdot \|_{1}}^{2}=\int_{0}^{1}\left(\left|f^{\prime}(x)\right|^{2}+|g(x)|^{2}\right) \mathrm{d} x+c_{1}|f(0)|^{2}, \forall(f, g) \in H,
$$

or

$$
\|(f, g)\|_{H ; \mid\|\|_{2}}^{2}=\int_{0}^{1}\left(\left|f^{\prime}(x)\right|^{2}+|g(x)|^{2}\right) \mathrm{d} x+c_{3}|f(0)|^{2}, \forall(f, g) \in H,
$$

where $c_{3}>0$. In the rest of this paper, we write norm $\|\cdot\|_{H}$ without discrimination.

Define the system operator $A: D(A) \rightarrow H$

$$
\left\{\begin{array}{l}
A(f, g)=\left(g, f^{\prime \prime}\right), \forall(f, g) \in D(A), \\
D(A)=\left\{(f, g) \in\left(H^{2}(0,1) \times H^{1}(0,1)\right) \mid A(f, g) \in H, f^{\prime}(0)=c_{0} g(0)+c_{1} f(0), f^{\prime}(1)=0\right\}
\end{array}\right.
$$

Then system (2.4) can be written as an abstract evolutionary equation in $H$.

$$
\frac{\mathrm{d}}{\mathrm{d} t}\left(\tilde{z}(\cdot, t), \tilde{z}_{t}(\cdot, t)\right)=A\left(\tilde{z}(\cdot, t), \tilde{z}_{t}(\cdot, t)\right)+B d(t),
$$

where $B(0, \delta(x-1))$ with $\delta(\cdot)$ being the Dirac distribution.

The following lemma is Lemma 2.1 in [19].

Lemma 2.1. Assume that $d \in L^{\infty}(0, \infty)$ or $d \in L^{2}(0, \infty)$. For any initial value $\left(\tilde{z}_{0}, \tilde{z}_{1}\right) \in H$, system (2.4) admits a unique bounded solution $\left(\tilde{z}(\cdot, t), \tilde{z}_{t}(\cdot, t)\right) \in C(0, \infty ; H)$.

Next, we design an observer for system (2.4)

$$
\left\{\begin{array}{l}
\theta_{t t}(x, t)=\theta_{x x}(x, t), x \in(0,1), t>0 \\
\theta_{x}(0, t)=c_{0} \theta_{t}(0, t)+c_{1} \theta(0, t), t \geq 0, \\
\theta_{x}(1, t)=\tilde{z}(1, t), t \geq 0, \\
\theta(x, 0)=\theta_{0}(x), \theta_{t}(x, 0)=\theta_{1}(x), x \in[0,1],
\end{array}\right.
$$

Notice that $\tilde{z}(1, t)=u(1, t)+w(1, t)+\beta(1, t)-\hat{z}(1, t)$ is known.

Let

$$
\begin{aligned}
r(x, t) & =\theta(x, t)-\tilde{z}(x, t)=\theta(x, t)-z(x, t)+\hat{z}(x, t) \\
& =\theta(x, t)-u(x, t)-w(x, t)-\beta(x, t)+\hat{z}(x, t)
\end{aligned} .
$$

Then $r(x, t)$ satisfies 


$$
\left\{\begin{array}{l}
r_{t t}(x, t)=r_{x x}(x, t), x \in(0,1), t>0 \\
r_{x}(0, t)=c_{0} r_{t}(0, t)+c_{1} r(0, t), t \geq 0, \\
r(1, t)=0, t \geq 0, \\
r(x, 0)=r_{0}(x), r_{t}(x, 0)=r_{1}(x), x \in[0,1],
\end{array}\right.
$$

Define the space $H_{E}^{1}(0,1)=\left\{f \in H^{1}(0,1) \mid f(1)=0\right\}$. Consider system (2.10) in the energy state space $H_{0}=H_{E}^{1}(0,1) \times L^{2}(0,1)$ with the norm

$$
\|(f, g)\|_{H_{0}}^{2}=\int_{0}^{1}\left(\left|f^{\prime}(x)\right|^{2}+|g(x)|^{2}\right) \mathrm{d} x, \forall(f, g) \in H_{0},
$$

Define the system operator $A_{0}: D\left(A_{0}\right) \rightarrow H_{0}$ for system (2.10) by

$$
\left\{\begin{array}{l}
A_{0}(f, g)=\left(g, f^{\prime \prime}\right), \forall(f, g) \in D\left(A_{0}\right), \\
D\left(A_{0}\right)=\left\{(f, g) \in\left(H^{2}(0,1) \times H^{1}(0,1)\right) \cap H_{0} \mid A_{0}(f, g) \in H_{0}, f^{\prime}(0)=c_{0} g(0)+c_{1} f(0)\right\}
\end{array}\right.
$$

Hence, system (2.10) can be written as an evolutionary equation in $H_{0}$ :

$$
\frac{\mathrm{d}}{\mathrm{d} t}\left(r(\cdot, t), r_{t}(\cdot, t)\right)=A_{0}\left(r(\cdot, t), r_{t}(\cdot, t)\right),
$$

Lemma 2.2. For any initial value $\left(r_{0}, r_{1}\right) \in H$, system (2.10) admits a unique solution $\left(r(\cdot, t), r_{t}(\cdot, t)\right) \in C\left(0, \infty ; H_{0}\right)$, which is exponentially stable in the sense that there exist two positive constants $L, \delta$ such that

$$
\left\|\left(r(\cdot, t), r_{t}(\cdot, t)\right)\right\|_{H_{0}} \leq L \mathrm{e}^{-\delta t}\left\|\left(r_{0}, r_{1}\right)\right\|_{H_{0}}, t \geq 0
$$

Moreover, $r_{x}(1, t) \in L^{2}(0, \infty)$ and there exists $M_{1}^{\prime}>0$ such that

$$
\int_{t_{1}}^{t_{2}} r_{x}^{2}(1, t) \mathrm{d} t \leq M_{1}^{\prime}\left(\mathrm{e}^{-2 \delta t_{1}}+\mathrm{e}^{-2 \delta t_{2}}\right), \forall t_{2}>t_{1}>0 .
$$

when the initial value $\left(r_{0}, r_{1}\right) \in D\left(A_{0}\right)$, the unique solution of system (2.10) satisfies $\left(r(\cdot, t), r_{t}(\cdot, t)\right) \in C\left(0, \infty ; D\left(A_{0}\right)\right)$ and $r_{x}(1, t)$ decays exponentially.

Proof. From [19] and [21], operator $A_{0}$ defined by (2.12) generates an exponentially stable $C_{0}$-semigroup $\mathrm{e}^{A_{0} t}$ on $H_{0}$. Hence for any initial value $\left(r_{0}, r_{1}\right) \in H$, system $(2.10)$ admits a unique solution $\left(r(\cdot, t), r_{t}(\cdot, t)\right) \in C\left(0, \infty ; H_{0}\right)$ and (2.14) holds.

Define Lyapunov functions for system (2.10)

$$
\begin{aligned}
& E_{v}(t)=\frac{1}{2} \int_{0}^{1}\left(r_{t}^{2}(x, t)+r_{x}^{2}(x, t)\right) \mathrm{d} x, \\
& p_{v}(t)=\int_{0}^{1}\left(x r_{t}(x, t) r_{x}(x, t)\right) \mathrm{d} x .
\end{aligned}
$$

Notice that $2 E_{v}(t)=\left\|\left(r(\cdot, t), r_{t}(\cdot, t)\right)\right\|_{H_{0}}^{2}$ decays exponentially and $\left|p_{v}(t)\right| \leq E_{v}(t)$. Differentiate $p_{v}(t)$ along the solution of system (2.10) to give

$$
\begin{aligned}
\dot{p}_{v}(t) & =\left.\frac{x}{2}\left(r_{t}^{2}(x, t)+r_{x}^{2}(x, t)\right)\right|_{0} ^{1}-\frac{1}{2} \int_{0}^{1}\left(r_{t}^{2}(x, t)+r_{x}^{2}(x, t)\right) \mathrm{d} x \\
& =\frac{1}{2} r_{x}^{2}(1, t)-E_{v}(t)
\end{aligned}
$$

Integrating above equation from $t_{1}$ to $t_{2}$ with respect to $t$, we have 


$$
\begin{aligned}
\int_{t_{1}}^{t_{2}} r_{x}^{2}(1, t) \mathrm{d} t & =2 \int_{t_{1}}^{t_{2}} E_{v}(t) \mathrm{d} t+2 p_{v}\left(t_{2}\right)-2 p_{v}\left(t_{1}\right) \\
& \leq \frac{2 L^{2} E_{v}(0)}{2 \delta}\left(\mathrm{e}^{-2 \delta t_{1}}-\mathrm{e}^{-2 \delta t_{2}}\right)+2 E_{v}\left(t_{2}\right)+2 E_{v}\left(t_{1}\right) \\
& \leq\left[\frac{L^{2} E_{v}(0)}{\delta}+2 L E_{v}(0)\right]\left(\mathrm{e}^{-2 \delta t_{1}}-\mathrm{e}^{-2 \delta t_{2}}\right),
\end{aligned}
$$

which implies (2.15) holds.

When the initial value $\left(r_{0}, r_{1}\right) \in D\left(A_{0}\right)$, we define a new variable $\bar{r}(x, t)=r_{t}(x, t)$. Then $\bar{r}(x, t)$ is governed by the following PDEs

$$
\left\{\begin{array}{l}
\bar{r}_{t t}(x, t)=\bar{r}_{x x}(x, t), x \in(0,1), t>0, \\
\bar{r}_{x}(0, t)=c_{0} \bar{r}_{t}(0, t)+c_{1} \bar{r}(0, t), t \geq 0, \\
\bar{r}(1, t)=0, t \geq 0, \\
\bar{r}(x, 0)=r_{1}(x), \bar{r}_{t}(x, 0)=r_{0}^{\prime \prime}(x), x \in[0,1] .
\end{array}\right.
$$

Notice that the initial value $\left(r_{1}, r_{0}^{\prime \prime}\right) \in H_{0}$. System (2.19) is the same as (2.10) except the initial value. From the first assertion, we conclude that

$$
\left\|\left(\bar{r}(\cdot, t), \bar{r}_{t}(\cdot, t)\right)\right\|_{H_{0}} \leq L \mathrm{e}^{-\delta t}\left\|\left(r_{1}, r_{0}^{\prime \prime}\right)\right\|_{H_{0}}, t \geq 0
$$

By Poincaré's inequality, it follows from the boundary condition of (2.10) at $x=0$ that

$$
\begin{aligned}
\left|r_{x}(1, t)\right|^{2} & \leq 2\left|r_{x}(0, t)\right|^{2}+2 \int_{0}^{1}\left|r_{x x}(x, t)\right|^{2} \mathrm{~d} x \\
& =2\left|c_{0} \bar{r}(0, t)+c_{1} r(0, t)\right|^{2}+2 \int_{0}^{1}\left|\bar{r}_{t}(x, t)\right|^{2} \mathrm{~d} x \\
& \leq 4 c_{0}^{2} \int_{0}^{1}\left|\bar{r}_{x}(x, t)\right|^{2} \mathrm{~d} x+4 c_{1}^{2} r_{0}^{2}+2 \int_{0}^{1}\left|\bar{r}_{t}(x, t)\right|^{2} \mathrm{~d} x \\
& \leq C^{\prime}\left(\left.\left\|\left.\left(r(\cdot, t), r_{t}(\cdot, t)\right)\right|_{H} ^{2}+\right\|\left(\bar{r}(\cdot, t), \bar{r}_{t}(\cdot, t)\right)\right|_{H_{0}} ^{2}\right)
\end{aligned}
$$

for some constant $C^{\prime}>0$. Hence $r_{x}(1, t)$ decays exponentially.

Remark 2.1. From Lemma 2.1, observer (2.9) is valid for system (2.4). Moreover, when $\left(r_{0}, r_{1}\right) \in D(A)$, we can obtain that $r_{x}(1, t) \rightarrow 0$ exponentially from Lemma 2.1. It equals to that $\theta_{x}(1, t) \rightarrow \tilde{z}_{x}(1, t)=d(t)$. Therefore, observer (2.9) can recover state and disturbance simultaneously for (2.4).

\section{Output Feedback Controller Design}

We design the output feedback of control for system (1.1) as follows

$$
U(t)=\left\{\begin{array}{l}
-\theta_{x}(1, t)+\frac{q+c_{0}}{1+c_{0}} u_{t}(0, t-1)-c_{3} u(1, t)+\frac{c_{3}\left(q+c_{0}\right)}{1+c_{0}} u(0, t-1), t>1 \\
-\theta_{x}(1, t)-c_{3} u(1, t), t<1
\end{array}\right.
$$

where $c_{3}>0$ is design parameter, $\theta_{x}(1, t), u_{t}(0, t-1), u(1, t)$ are given by the system (2.9) and (1.1) respectively.

Under the controller (3.1), next considering the case of $t>1$, the closed-loop system (1.1) can be written as 


$$
\left\{\begin{array}{l}
u_{t t}(x, t)=u_{x x}(x, t), \\
u_{x}(0, t)=-q u_{t}(0, t), \\
u_{x}(1, t)=d(t)-\theta_{x}(1, t)+\frac{q+c_{0}}{1+c_{0}} u_{t}(0, t-1)-c_{3} u(1, t)+\frac{c_{3}\left(q+c_{0}\right)}{1+c_{0}} u(0, t-1), \\
w_{x}(x, t)+w_{x}(x, t)=0, \\
w(0, t)=-\frac{q+c_{0}}{1+c_{0}} u(0, t), \\
\beta_{t t}(x, t)=\beta_{x x}(x, t), \\
\beta_{x}(0, t)=c_{0} \beta_{t}(0, t)+c_{1} \beta(0, t)+c_{1} u(0, t)+c_{1} w(0, t), \\
\beta_{x}(1, t)=0, \\
\hat{z}_{t t}(x, t)=\hat{z}_{x x}(x, t), \\
\hat{z}_{x}(0, t)=c_{0} \hat{z}_{t}(0, t)+c_{1} \hat{z}(0, t), \\
\hat{z}_{x}(1, t)=-\theta_{x}(1, t)-c_{3} u(1, t)+\frac{c_{3}\left(q+c_{0}\right)}{1+c_{0}} u(0, t-1), \\
\theta_{t t}(x, t)=\theta_{x x}(x, t), \\
\theta_{x}(0, t)=c_{0} \theta_{t}(0, t)+c_{1} \theta(0, t), \\
\theta(1, t)=u(1, t)-\frac{c_{3}\left(q+c_{0}\right)}{1+c_{0}} u(0, t-1)+\beta(1, t)-\hat{z}(1, t),
\end{array}\right.
$$

where the initial value of system (3.2) is $\left(u_{0}, u_{1}, w_{0}, \beta_{0}, \beta_{1}, \hat{z}_{0}, \hat{z}_{1}, \theta_{0}, \theta_{1}\right)$.

Consider system (3.2) in the state space

$$
\begin{aligned}
H^{\prime}=\{ & \left(f_{1}, g_{1}, f_{2}, f_{3}, g_{3,} f_{4}, g_{4}, f_{5}, g_{5}\right) \in H^{1}(0,1) \times L^{2}(0,1) \times H^{1}(0,1) \\
\times & \left(H^{1}(0,1) \times L^{2}(0,1)\right)^{3} \mid f_{2}(0)=\frac{q+c_{0}}{1+c_{0}} f_{1}(0) \\
& \left.f_{5}(1)=f_{1}(1)+f_{2}(1)+f_{3}(1)-f_{4}(1)\right\}
\end{aligned}
$$

with the norm

$$
\begin{aligned}
& \left\|\left(f_{1}, g_{1}, f_{2}, f_{3}, g_{3}, f_{4}, g_{4}, f_{5}, g_{5}\right)\right\|_{H^{\prime}}^{2} \\
& =\int_{0}^{1}\left(\left|f_{1}^{\prime}(x)\right|^{2}+\left|g_{1}(x)\right|^{2}+\left|f_{2}^{\prime}(x)\right|^{2}+\left|f_{3}^{\prime}(x)\right|^{2}+\left|g_{3}(x)\right|^{2}\right. \\
& \left.\quad+\left|f_{4}^{\prime}(x)\right|^{2}+\left|g_{4}(x)\right|^{2}+\left|f_{5}^{\prime}(x)\right|^{2}+\left|g_{5}(x)\right|^{2}\right) \mathrm{d} x \\
& \quad+c_{3}\left|f_{1}(1)\right|^{2}+\left|f_{2}(0)\right|^{2}+c_{1}\left|f_{3}(0)\right|^{2}+c_{1}\left|f_{4}(0)\right|^{2}
\end{aligned}
$$

Theorem 3.1. Assume that $c_{0}, c_{1}, c_{3}>0$, For any initial value $\left(u_{0}, u_{1}, w_{0}, \beta_{0}, \beta_{1}, \hat{z}_{0}, \hat{z}_{1}, \theta_{0}, \theta_{1}\right) \in H^{\prime}$, System (3.2) admits a unique bounded solution

$\left(u(\cdot, t), u_{t}(\cdot, t), w(\cdot, t), \beta(\cdot, t), \beta_{t}(\cdot, t), \hat{z}(\cdot, t), z_{t}(\cdot, t), \theta(\bullet, t), \theta_{t}(\cdot, t)\right) \in C\left(0, \infty ; H^{\prime}\right)$.

Moreover, the solution of u-subsystem is exponentially stable in the sense that there exist two positive constants $M, \varepsilon$, such that

$$
\left\|u(\cdot, t), u_{t}(\cdot, t)\right\|_{H} \leq M \mathrm{e}^{-\varepsilon t}\left\|\left(u_{0}, u_{1}, w_{0}, \beta_{0}, \beta_{1}, \hat{z}_{0}, \hat{z}_{1}, \theta_{0}, \theta_{1}\right)\right\|_{H}, \forall t \geq 0
$$

Proof. Introducing a new variable $\gamma(x, t)=u(x, t)+w(x, t)$ and the variables 
$\tilde{z}(x, t), r(x, t)$ in Section 2 , that is,

$$
\left(\begin{array}{l}
\gamma \\
w \\
\beta \\
\tilde{z} \\
r
\end{array}\right)=\left(\begin{array}{ccccc}
1 & 1 & 0 & 0 & 0 \\
0 & 1 & 0 & 0 & 0 \\
0 & 0 & 1 & 0 & 0 \\
1 & 1 & 1 & -1 & 0 \\
-1 & -1 & -1 & 1 & 1
\end{array}\right)\left(\begin{array}{l}
u \\
w \\
\beta \\
\hat{z} \\
\theta
\end{array}\right),
$$

we can transform system (3.2) into the following equivalent system

$$
\left\{\begin{array}{l}
\gamma_{t t}(x, t)=\gamma_{x x}(x, t), \\
\gamma_{x}(0, t)=c_{0} \gamma_{t}(0, t), \\
\gamma_{x}(1, t)=-c_{3} \gamma(1, t)-r_{x}(1, t), \\
w_{x}(x, t)+w_{x}(x, t)=0, \\
w(0, t)=-\frac{q+c_{0}}{1-q} \gamma(0, t), \\
\beta_{t t}(x, t)=\beta_{x x}(x, t), \\
\beta_{x}(0, t)=c_{0} \beta_{t}(0, t)+c_{1} \beta(0, t)+c_{1} \gamma(0, t), \\
\beta_{x}(1, t)=0, \\
\tilde{z}_{t t}(x, t)=\tilde{z}_{x x}(x, t), \\
\tilde{z}_{x}(0, t)=c_{0} \tilde{z}_{t}(0, t)+c_{1} \tilde{z}(0, t), \\
\tilde{z}_{x}(1, t)=d(t), \\
r_{t t}(x, t)=r_{x x}(x, t), \\
r_{x}(0, t)=c_{0} r_{t}(0, t)+c_{1} r(0, t), \\
r(1, t)=0 .
\end{array}\right.
$$

where the initial value

$$
\left\{\begin{array}{l}
\gamma(x, 0)=\gamma_{0}(x)=u_{0}(x)+w_{0}(x), \gamma_{t}(x, 0)=\gamma_{1}(x)=u_{1}(x)-w_{0}^{\prime}(x), \\
w(x, 0)=w_{0}(x), \beta(x, 0)=\beta_{0}(x), \beta_{t}(x, 0)=\beta_{1}(x) \\
\tilde{z}(x, 0)=\tilde{z}_{0}(x)=u_{0}(x)+w_{0}(x)+\beta_{0}(x)-\hat{z}_{0}(x) \\
\tilde{z}_{t}(x, 0)=\tilde{z}_{1}(x)=u_{1}(x)+w_{0}^{\prime}(x)+\beta_{1}(x)-\hat{z}_{1}(x) \\
r(x, 0)=r_{0}(x)=\theta_{0}(x)+\hat{z}_{0}(x)-u_{0}(x)-w_{0}(x)-\beta_{0}(x) \\
r_{t}(x, 0)=r_{1}(x)=\theta_{1}(x)+\hat{z}_{1}(x)-u_{1}(x)-w_{0}^{\prime}(x)-\beta_{1}(x) .
\end{array}\right.
$$

We will consider the $r$-subsystems of (3.7) respectively. For any initial value $\left(u_{0}, u_{1}, w_{0}, \beta_{0}, \beta_{1}, \hat{z}_{0}, \hat{z}_{1}, \theta_{0}, \theta_{1}\right) \in H^{\prime}$, we have $\left(\gamma_{0}, \gamma_{1}, w_{0}, \beta_{0}, \beta_{1}, \hat{z}_{0}, \hat{z}_{1}, r_{0}, r_{1}\right) \in H \times H^{1}(0,1) \times H^{2} \times H_{0}$.

Since $\left(r_{0}, r_{1}\right) \in H_{0}$, the $\tilde{z}$-subsystem of (3.7) admits a unique exponentially stable solution $\left(r(\cdot, t), r_{t}(\cdot, t)\right) \in C\left(0, \infty ; H_{0}\right)$ guaranteed by Lemma 2.2 .

Since $\left(\tilde{z}_{0}, \tilde{z}_{1}\right) \in H$, the -subsystem of (3.7) admits a unique exponentially stable solution $\left(\tilde{z}(\cdot, t), \tilde{z}_{t}(\bullet, t)\right) \in C(0, \infty ; H)$ guaranteed by Lemma 2.1.

Define the system operator $A_{1}: D\left(A_{1}\right) \rightarrow H$

$$
\left\{\begin{array}{l}
A_{1}(f, g)=\left(g, f^{\prime \prime}\right), \forall(f, g) \in D\left(A_{1}\right), \\
D\left(A_{1}\right)=\left\{(f, g) \in\left(H^{2}(0,1) \times H^{1}(0,1)\right) \mid A(f, g) \in H, f^{\prime}(0)=c_{0} g(0), f^{\prime}(1)=-c_{3} f(1)\right\} .
\end{array}\right.
$$


Then the $\gamma$-subsystem can be written as an abstract evolutionary equation in H

$$
\frac{\mathrm{d}}{\mathrm{d} t}\left(\gamma(\cdot, t), \gamma_{t}(\cdot, t)\right)=A_{1}\left(\gamma(\cdot, t), \gamma_{t}(\cdot, t)\right)+B_{1}\left(-r_{x}(1, t)\right)
$$

where $B_{1}=(0, \delta(x-1))$.

It is well-known that operator $A_{1}$ generates an exponentially stable $C_{0}$ -semigroup $\mathrm{e}^{A_{1} t}$ on $H$. It is a routine exercise that $B_{1}$ is admissible for $\mathrm{e}^{A_{1} t}$ ([22]). Then the solution to system (3.9) can be written as

$$
\left(\gamma(\cdot, t), \gamma_{t}(\cdot, t)\right)=\mathrm{e}^{A_{1} t}\left(\gamma_{0}, \gamma_{1}\right)+\int_{0}^{t} \mathrm{e}^{A_{1}(t-s)} B_{1}\left(-\gamma_{x}(1, s)\right) \mathrm{d} s .
$$

From (2.15) in Lemma 2.2 and the estimation in

$$
\left\|\int_{0}^{t} \mathrm{e}^{A(t-s)} B_{1} \varepsilon_{s}(0, s) \mathrm{d} s\right\|_{H}^{2} \leq 2 M_{1}^{2} \mathrm{e}^{-\beta t} K_{t} \frac{M_{2}}{k_{1}} E_{\varepsilon}(0)\left(1+\mathrm{e}^{-\frac{\alpha_{2} t}{2}}\right)+4 K_{1} \frac{M_{2}}{k_{1}} E_{\varepsilon}(0) \mathrm{e}^{-\frac{\alpha_{2} t}{2}}
$$

(61) of [23], we obtain the exponential stability of $\gamma$-subsystem in the sense that there exist two positive constants $M_{2}, \delta_{2}$ such that

$$
\left\|\gamma(\cdot, t), \gamma_{t}(\cdot, t)\right\|_{H} \leq M_{2} \mathrm{e}^{-\delta_{2} t}\left\|\left(\gamma_{0}, \gamma_{1}\right)\right\|_{H}, \forall t \geq 0 .
$$

Define Lyapunov functions for $\gamma$-subsystem

$$
\begin{aligned}
& E_{\gamma}(t)=\frac{1}{2} \int_{0}^{1}\left(\gamma_{t}^{2}(x, t)+\gamma_{x}^{2}(x, t)\right) \mathrm{d} x+\frac{c_{3}}{2}|\gamma(1, t)|^{2}, \\
& p_{\gamma}(t)=\int_{0}^{1} x \gamma_{t}(x, t) \gamma_{x}(x, t) \mathrm{d} x .
\end{aligned}
$$

Notice that $2 E_{\gamma} t=\left\|\gamma(\cdot, t), \gamma_{t}(\cdot, t)\right\|_{H}^{2}$ decays exponentially and $\left|p_{\gamma}(t)\right| \leq E_{\gamma}(t)$. Differentiate $p_{\gamma}(t)$ along the solution of $\gamma$-subsystem to give

$$
\begin{aligned}
\dot{p}_{\gamma}(t) & =\left.\frac{x-1}{2}\left(\gamma_{x}^{2}(x, t)+\gamma_{t}^{2}(x, t)\right)\right|_{0} ^{1}-\frac{1}{2} \int_{0}^{1}\left(\gamma_{t}^{2}(x, t)+\gamma_{x}^{2}(x, t)\right) \mathrm{d} x \\
& =\frac{1}{2}\left(\gamma_{x}^{2}(0, t)+\gamma_{t}^{2}(0, t)\right)-\frac{1}{2} \int_{0}^{1}\left(\gamma_{t}^{2}(x, t)+\gamma_{x}^{2}(x, t)\right) \mathrm{d} x
\end{aligned}
$$

Integrating above equation from $t-1$ to $t$, we have

$$
\begin{aligned}
& \int_{t-1}^{t}\left(\gamma_{x}^{2}(0, s)+\gamma_{t}^{2}(0, s)\right) \mathrm{d} s \\
& \leq 2 \int_{t-1}^{t} E_{\gamma}(s) \mathrm{d} s+2 p_{\gamma}(t)-2 p_{\gamma}(t-1) \\
& \leq \frac{2 M_{2}^{2} E_{\gamma}(0)}{2 \delta_{2}}\left(\mathrm{e}^{-2 \delta_{2}(t-1)}-\mathrm{e}^{-2 \delta_{2} t}\right)+2 E_{\gamma}(t)+2 E_{\gamma}(t-1) \\
& \leq\left[\frac{M_{2}^{2} E_{\gamma}(0)}{\delta_{2}} \mathrm{e}^{2 \delta_{2}}+2 M_{2} E_{\gamma}(0)+2 M_{2} E_{\gamma}(0) \mathrm{e}^{2 \delta_{2}}\right] \mathrm{e}^{-2 \delta_{2} t}
\end{aligned}
$$

For the $w$-subsystem, the exact solution can be written as

$$
w(x, t)=\left\{\begin{array}{l}
w_{0}(x-t), x \geq t, \\
-\frac{q+c_{0}}{1-q} \gamma(0, t-x), t \geq x .
\end{array}\right.
$$

which has the estimation 


$$
\begin{aligned}
\int_{0}^{1}\left(\left|w_{t}(x, t)\right|^{2}+\left|w_{x}(x, t)\right|^{2}\right) \mathrm{d} x & \leq \frac{2\left(q+c_{0}\right)^{2}}{(1-q)^{2}} \int_{0}^{1}\left|\gamma_{t}(0, t-x)\right|^{2} \mathrm{~d} x \\
& =\frac{2\left(q+c_{0}\right)^{2}}{(1-q)^{2}} \int_{t-1}^{t}\left|\gamma_{t}(0, s)\right|^{2} \mathrm{~d} s, t \geq 1 \\
|w(0, t)|^{2} & =\frac{\left(q+c_{0}\right)^{2}}{(1-q)^{2}}|\gamma(0, t)|^{2}
\end{aligned}
$$

From (3.12) and (3.15), $\left\|w(\cdot, t), w_{t}(\cdot, t)\right\|_{H}$ decays exponentially.

For $\beta$-subsystem, we can write it into an abstract evolutionary equation in $H$.

$$
\frac{\mathrm{d}}{\mathrm{d} t}\left(\beta(\cdot, t), \beta_{t}(\cdot, t)\right)=A\left(\beta(\cdot, t), \beta_{t}(\cdot, t)\right)+B_{3}\left(-c_{3} \gamma(0, t)\right),
$$

where $A$ is defined by (2.7) and $B_{3}=(0, \delta(x))$. Direct computations indicate that $B_{3}$ is admissible for $\mathrm{e}^{A t}$. Hence the solution to (3.18) can be written as

$$
\left(\beta(\cdot, t), \beta_{t}(\cdot, t)\right)=\mathrm{e}^{A t}\left(\beta_{0}, \beta_{1}\right)+\int_{0}^{t} \mathrm{e}^{A(t-s)} B_{3}\left(-c_{3} \gamma(0, s)\right) \mathrm{d} s,
$$

which is exponentially stable because of the exponential decay of $\gamma(0, t)$. Hence we obtain that system (3.7) admits a unique bounded solution

By the bounded inverse transformation of (3.6), we obtain the solution to system (3.2) as follows

$$
\left\{\begin{array}{l}
u(x, t)=\gamma(x, t)-w(x, t), u_{t}(x, t)=\gamma_{t}(x, t)-w_{t}(x, t), \\
w(x, t)=w(x, t), \beta(x, t)=\beta(x, t), \beta_{t}(x, t)=\beta_{t}(x, t), \\
\hat{z}(x, t)=\gamma(x, t)+\beta(x, t)-\hat{z}(x, t), \hat{z}_{t}(x, t)=\gamma_{t}(x, t)+\beta_{t}(x, t)-\hat{z}_{t}(x, t), \\
\theta(x, t)=r(x, t)+\tilde{z}(x, t), \theta_{t}(x, t)=r_{t}(x, t)+\tilde{z}_{t}(x, t) .
\end{array}\right.
$$

Hence system (3.2) admits a unique bounded solution. From the exponential stability of $\gamma$ - and $w$-subsystems, $u$-subsystem is exponentially stable.

\section{Conclusion Remarks}

In this paper, the anti stable wave equation with disturbance is controlled. Firstly, the disturbance estimator is designed for the original system, and then the disturbance is estimated. Then, the controller is designed to realize the control. In addition, many references, especially the relevant data about ADRC, are used to verify the closed-loop system, the closed-loop system is simplified and the content of this paper is obtained.

\section{Conflicts of Interest}

The author declares no conflicts of interest regarding the publication of this paper.

\section{References}

[1] Guo, B.Z. and Kang, W. (2014) The Lyapunov Approach to Boundary Stabilization of an Anti-Stable One-Dimensional Wave Equation with Boundary Disturbance. International Journal of Robust and Nonlinear Control, 24, 54-69. 
https://doi.org/10.1002/rnc.2874

[2] Zhao, Z.L. and Guo, B.Z. (2015) On Active Disturbance Rejection Control for Nonlinear Systems Using Time-Varying Gain. European Journal of Control, 23, 62-70. https://doi.org/10.1016/j.ejcon.2015.02.002

[3] Wu, Z.H. and Guo, B.Z. (2020) Active Disturbance Rejection Control to MIMO Nonlinear Systems with Stochastic Uncertainties: Approximate Decoupling and Output-Feedback Stabilization. International Journal of Control, 93, 1408-1427.

[4] Guo, B.Z. and Zhou, H.C. (2015) The Active Disturbance Rejection Control to Stabilization for Multi-Dimensional Wave Equation with Boundary Control Matched Disturbance. IEEE Transactions on Automatic Control, 60, 143-157. https://doi.org/10.1109/TAC.2014.2335511

[5] Guo, B.Z. and Zhou, H.C. (2014) Active Disturbance Rejection Control for Rejecting Boundary Disturbance from Multi-Dimensional Kirchhoff Plate via Boundary Control. SIAM Journal on Control and Optimization, 52, 2800-2830. https://doi.org/10.1137/130940918

[6] Zhao, Z.L. and Guo, B.Z. (2015) Extended State Observer for Uncertain Lower Triangular Nonlinear Systems. Systems and Control Letters, 85, 100-108. https://doi.org/10.1016/j.sysconle.2015.09.004

[7] Guo, B.Z. and Zhao, Z.L. (2011) On the Convergence of Extended State Observer for Nonlinear Systems with Uncertainty. Systems and Control Letters, 60, 420-430. https://doi.org/10.1016/j.sysconle.2011.03.008

[8] Zhao, Z.L. and Guo, B.Z. (2017) A Nonlinear Extended State Observer Based on Fractional Power Functions. Automatica, 81, 286-296. https://doi.org/10.1016/j.automatica.2017.03.002

[9] Wu, Z.H. and Guo, B.Z. (2016) Extended State Observer for Uncertain Lower Triangular Nonlinear Systems Subject to Stochastic Disturbance. Control Theory and Technology, 14, 179-188. https://doi.org/10.1007/s11768-016-6019-4

[10] Guo, B.Z. and Jin, F.F. (2015) Output Feedback Stabilization for One-Dimensional Wave Equation Subject to Boundary Disturbance. IEEE Transactions on Automatic Control, 60, 824-830. https://doi.org/10.1109/TAC.2014.2335374

[11] Waiker, J.A. (1980) Dynamical Systems and Evolution Equation: Theory and Applications. Plenum Press, New York.

[12] Guo, W. and Guo, B.Z. (2013) Stabilization and Regulator Design for a One-Dimensional Unstable Wave Equation with Input Harmonic Disturbance. International Journal of Robust and Nonlinear Control, 23, 514-533. https://doi.org/10.1002/rnc. 1843

[13] Zhou, H.C. and Guo, B.Z. (2018) Performance Output Tracking for One-Dimensional Wave Equation Subject to Unmatched General Disturbance and Non-Collocated Control. European Journal of Control, 39, 39-52. https://doi.org/10.1016/j.ejcon.2017.10.006

[14] Guo, B.Z., Zhou, H.C., Al-Fhaid, A.S., Younas, A.M.M. and Asiri, A. (2015) Parameter Estimation and Stabilization for One-Dimensional Schrodinger Equation with Boundary Output Constant Disturbance and Non-Collocated Equation with Boundary Output Constant Disturbance and Non-Collocated Control. Journal of the Franklin Institute, 352, 2047-2064. https://doi.org/10.1016/j.jfranklin.2015.02.020

[15] Guo, B.Z. and Liu, J.J. (2014) Sliding Mode Control and Active Disturbance Rejection Control to the Stabilization of One-Dimensional Schrodinger Equation Subject to Boundary Control Matched Disturbance. International Journal of Robust and 
Nonlinear Control, 24, 2194-2212. https://doi.org/10.1002/rnc.2977

[16] Guo, B.Z. and Jin, F.F. (2013) Sliding Mode and Active Disturbance Rejection Control to the Stabilization of Anti-Stable One-Dimensional Wave Equation Subject to Boundary Input Disturbance. IEEE Transactions on Automatic Control, 58, 1269-1274. https://doi.org/10.1109/TAC.2012.2218669

[17] Guo, B.Z. and Jin, F.F. (2013) The Active Disturbance Rejection and Sliding Mode Control Approach to the Stabilization of Euler-Bernoulli Beam Equation with Boundary Input Disturbance. Automatica, 49, 2911-2918.

https://doi.org/10.1016/j.automatica.2013.06.018

[18] Chauvin, J. (2011) Observer Design for a Class of Wave Equation Driven by an Unknown Periodic Input. 18th IFAC World Congress, Italy, 1332-1337.

[19] Feng, H. and Guo, B.Z. (2017) A New Active Disturbance Rejection Control to Output Feedback Stabilization for a One-Dimensional Anti-Stable Wave Equation with Disturbance. IEEE Transactions on Automatic Control, 62, 3774-3787. https://doi.org/10.1109/TAC.2016.2636571

[20] Zhou, H.-C. and Weiss, G. (2017) Output Feedback Exponential Stabilization of a Nonlinear 1-D Wave Equation with Boundary Input. IFAC-PapersOnLine, 50, 5586-5591. https://doi.org/10.1016/j.ifacol.2017.08.1103

[21] Chen, G. (1981) A Note on the Boundary Stabilization of the Wave Equation. SIAM Journal on Control and Optimization, 19, 106-113. https://doi.org/10.1137/0319008

[22] Weiss, G. (1989) Admissibility of Unbounded Control Operators. SIAM Journal on Control and Optimization, 27, 527-545. https://doi.org/10.1137/0327028

[23] Su, L., Guo, W., Wang, J. and Krstic, M. (2017) Boundary Stabilization of Wave Equation with Velocity Recirculation. IEEE Transactions on Automatic Control, 62, 4760-4767. https://doi.org/10.1109/TAC.2017.2688128 